\title{
Administrando el cambio
}

\section{Managing the change}

Eduardo X. Jurado Vinueza

Pontificia Universidad Católica del Perú, Perú

Autor para correspondencia: ejuradov@ pucp.pe

Fecha de recepción: 20 de enero de 2016 - Fecha de aceptación: 20 de febrero de 2016

\section{Resumen}

Este estudio analiza el efecto que tienen los cambios en las empresas y en las vidas de los empleados. Examina de donde vienen los cambios disruptivos y ofrece algunas sugerencias para líderes de empresas para que anticipen cambios y hagan planes para poder ser flexibles cuando vengan tales cambios.

Palabras claves: cambios; plan de negocio; nuevas estrategias

\begin{abstract}
This study analyzes the effects that changes have in companies and in the lives of employees. It examines where disruptive changes come from and offers suggestions as to how company leaders can anticípate changes and make plans in order to be flexible when such changes come.
\end{abstract}

Key words: changes; business plans; new strategies' 


\section{Introducción}

Gran parte de la turbulencia en los empleos son dados por factores fuera del control personal y hasta de las empresas lo cual afecta profundamente el modelo de negocio que las organizaciones usan, generándose así nuevos modelos los cuales generan cambios disruptivos creando nuevas oportunidades para ciertos trabajadores y desempleo para otros (T. Clark, Osterwalder, \& Pigneur, 2012). Una efectiva administración del cambio es importante para el desarrollo organizacional, pero desconocer las características que impactan al empleado en una situación de cambio, crea dificultad en administrar el cambio exitosamente (Lattuch \& Young, 2011).

En un mundo competitivo donde la tecnología crea constantemente cambios disruptivos, las organizaciones deben tener la flexibilidad de anticiparse y adaptarse a dichos cambios los cuales llevan a la desaparición de productos o servicios. Para que una organización sea exitosa, sus sistemas y prácticas deben ser flexibles no sólo para apoyar sus objetivos de negocio, sino también para atender a las necesidades fisiológicas y mentales de sus miembros ("Theoretical Foundation for Sociocultural Homeostasis," 2012).

Las organizaciones están compuestas por personas. Las personas y los grupos son sistemas complejos adaptativos gobernados por la auto-organización no por el poder del cargo (Ehin, 2013). Una administración efectiva del cambio es importante para el desarrollo organizacional, pero se debe tener en cuenta que factores del cambio impactan en el comportamiento del empleado (Lattuch \& Young, 2011). Los parámetros de una organización pueden ser ajustados o administrados, pero no se puede precisar el resultado del comportamiento de las personas dentro de esta estructura (Ehin, 2009).

Las organizaciones homeostáticas se adaptan rápidamente a dichos cambios logrando así su supervivencia, generando, paralelamente mayor competitividad ya que su principio se basa en el equilibrio dinámico donde todas las partes se desarrollan al mismo nivel, tal como si la empresa fuese un gran organismo vivo.

\section{Revisión de la literatura}

Debido a los cambios disruptivos promovidos por la innovación y la tecnología, es imperativo recordar que las organizaciones no están compuestas por estructuras artificiales que típicamente son visualizadas y organizadas por la administración, sino que están compuestas por redes sociales las cuales evolucionan constantemente. Estas redes emergentes son entidades orgánicas y auto-organizadas las cuales pueden ser influenciadas, pero no controladas (Ehin \& Kivikas, 2012). La frecuencia de cambio y la planificación para el cambio están significativamente relacionados con la incertidumbre, ya su vez, que la incertidumbre está significativamente relacionada con la satisfacción laboral y el estrés conductual (Lattuch \& Young, 2011).

Muchas de las escuelas de negocios, continúan centradas en las cuatro funciones de gestión - planificación, organización, dirección y control - las cuales son construcciones artificiales que proveen de poca ayuda al invisible dinamismo de gestión social y sus sistemas 
emergentes (Ehin, 2013). Para que una organización sea exitosa, sus sistemas y prácticas deben ser flexibles no sólo para apoyar sus objetivos de negocio, sino también para atender a las necesidades fisiológicas y mentales de sus miembros ("Theoretical Foundation for Sociocultural Homeostasis," 2012).

La teoría económica moderna ignora la influencia de emociones en la toma de decisiones, por otro lado, la evidencia emergente de la neurociencia, sugiere que la toma de decisiones racional depende de un previo y preciso procesamiento emocional (Bechara \& Damasio, 2005). Adicionalmente, las investigaciones demuestran que las emociones llevan a tomar mejores decisiones que la lógica (Jacobs, 2009). Las nuevas y actuales teorías del comportamiento organizacional pueden ser mejoradas incorporando hallazgos de la neurociencia basado en como el cerebro produce cognición, actitudes y comportamientos (Becker \& Cropanzano, 2010). Las organizaciones pueden ser consideradas saludables si sus miembros observan ciertos códigos no declarados de comportamiento que se aceptan como normales, los cuales permite a todos los niveles de la organización cumplir con el status quo y el crecimiento (J. V. Clark, 1962) por lo tanto, lo que piensan los empleados cuando están dentro del trabajo afecta las relaciones entre una organización basada en la autoestima, y las percepciones y actitudes de los empleados hacia su lugar de trabajo (Gardner \& Pierce, 2013).

Los sistemas orientados a metas habituales del cerebro tienden a estabilizar, en torno a puntos homeostáticos, el estado del cuerpo y de la mente cuyo mantenimiento produce satisfacción o felicidad (Camerer, Loewenstein, \& Prelec, 2005). La desviación en torno a estos puntos, crea malestar, emociones negativas, e incluso dolor por lo que regresar a esta zona de confort genera satisfacción y emoción positiva, por lo tanto, la homeostasis y el sistema de toma de decisiones basado en hábitos, pueden trabajar en contra de las metas racionales propuestas por el sistema de planeación (Becker \& Cropanzano, 2010). Tanto las variedades homeostáticas básicas y socioculturales promueven el mismo objetivo, que es la supervivencia de los organismos vivos; en el caso del homeostasis sociocultural, ese objetivo se amplía abarcando la búsqueda deliberada del bienestar. (Damasio, 2010).

Es importante recordar que no existen dos personas que son psicológicamente iguales, por lo que antes de tomar alguna acción, los individuos interpretarán cada situación (proceso, problema, oportunidad o clima laboral) a su propia manera (Ehin, 2010). La existencia del conocimiento con dominio-específico sugiere que las personas parecen ser genios para ciertas tareas, pero si tratan con tareas superficialmente distintas, pasaría lo contrario, por lo cual el procesamiento de este dominio-específico tiene implicaciones importantes para la economía y en especial para las organizaciones laborales (Camerer et al., 2005). Uno de los obstáculos más difíciles para el cambio organizacional, es la superación de la resistencia individual al cambio; aunque los empleados indican que están a favor, a menudo su comportamiento subvierte el cambio (Becker \& Cropanzano, 2010). La agrupación de estas tareas en puestos de trabajo requiere comprender cuales son las habilidades generales útiles para cualquier tarea, y cuales son las habilidades que son separadas neurológicamente (Camerer et al., 2005).

La "homeóstasis sociocultural", la cual es creada y guiada por la conciencia, opera como un sanador con valor biológico, la cual promueve la supervivencia de los organismos vivos (Damasio, 2010). Los economistas más influyentes usualmente están abiertos a explicaciones 
sobre como los humanos actúan, creando modelos de comportamiento humano las cuales incluyen nociones tales como principios o normas sociales (por ejemplo, modelos evolucionarios de teoría de juego donde las estrategias son frecuentemente interpretadas como normas sociales o como otros modelos de interacción social) (Martins, 2010). Dentro de las empresas, los cuatro factores dinámicos fundamentales que impactan la homeostasis sociocultural son la auto organización, el tamaño del grupo, la dinámica social y el "punto dulce de la organización” (Ehin, 2009, 2013).

Basado en el marco general de los cuatro factores que impactan la homeostasis sociocultural como una constante de evolución dinámica, permite aumentar la participación de los trabajadores, la productividad y la innovación de dentro de las empresas (Ehin, 2013), se plantean las siguientes hipótesis:

- H1: La homeostasis organizacional es importante para el incremento de la productividad y la capacidad de innovar de las empresas.

- H2: La homeostasis organizacional tiene un impacto significativamente positivo para una efectiva administración del cambio.

Los líderes y gerentes deben tener en consideración la importancia del balance homeostático a largo plazo del planeta y deben creer que la naturaleza y los humanos conforman un ecosistema y que las organizaciones tienen el rol de mantener y mejorar dichos ecosistemas (Parkes \& Borland, 2012). Las relaciones son una importante parte de la naturaleza humana y uno de los componentes más críticos para el incremento de la productividad e innovación (Cacioppo \& Patrick, 2008).

La vida es un acto de balanceo constante y dinámico tanto a nivel grupal como de individuo, ergo para que una empresa sea exitosa, sus sistemas y prácticas deben ser flexibles no solo para soportar las metas del negocio sino también las necesidades fisiológicas y mentales de sus miembros (Ehin, 2013). La regulación de la vida, un proceso dinámico conocido como homeostasis, tiene sus inicios desde organismos vivientes unicelulares los cuales no tienen cerebro, pero tienen un comportamiento adaptativo (Damasio, 2010). El término homeostasis es comúnmente conocido como la tendencia biológica de una entidad para mantener su estabilidad interna basada en la reacción coordinada de sus componentes sobre una situación o estímulo que afecte su función o condición normal (Ehin, 2013).

Para comprender como los sistemas afectivos operan, debemos recalcar que los humanos no evolucionaron para ser felices, sino para sobrevivir y reproducirse por lo que un importante proceso que el cuerpo realiza para alcanzar estos objeticos es la homeostasis (Camerer et al., 2005). La homeostasis organizacional es basada en soportar las necesidades biológicas y psicológicas de las personas, más que en la restricción artificial de estas (Ehin, 2013).

Los economistas están acostumbrados a pensar que las preferencias son el punto de partida del comportamiento humano y que el comportamiento es el punto final. En contraste, la neurociencia usa comportamientos explícitos como uno de los múltiples mecanismos que el cerebro utiliza para mantener su homeostasis, y preferencias como variables de estado 
transitorios que garantizan la supervivencia y reproducción (Camerer et al., 2005). La homeostasis, en el sentido de mantener su forma estructural bastantemente constante, es un elemento importante para la sobrevivencia de la organización (Butts \& Carley, 2007) por lo que si esta no es capaz de mantener un estado de homeostasis por períodos largos de tiempo, corre el riesgo de incurrir costos que inducen a su mortalidad (Hannan \& Freeman, 1977).

\section{Conclusión}

Las organizaciones están constantemente generando nuevos modelos de negocio para mantenerse competitivas, debido a externalidades. Estos nuevos modelos crean cambios disruptivos creando nuevas oportunidades de trabajo para unos y desempleo para otros (T. Clark et al., 2012). Debido a la importancia de la innovación en el ambiente actual, es imperativo recordar que las organizaciones están compuestas por redes sociales que evolucionan constantemente y no por estructuras artificiales típicamente visualizadas y organizadas por la administración (Ehin \& Kivikas, 2012). Las organizaciones están compuestas por personas que son impulsivas acerca de sus metas, sentimientos y acciones, con la frecuencia de al menos cuando son racionales (Ehin, 2009). Adicionalmente, estas redes sociales emergentes, no son máquinas, son entidades orgánicas y auto-organizadas las cuales pueden ser influenciadas pero no controladas (Ehin \& Kivikas, 2012).

La búsqueda del homeostasis organizacional es importante para tanto el incremento de la productividad y capacidad de innovación de la empresa como para el bienestar físico y mental de los trabajadores y de la sociedad (Ehin, 2013). El incremento de la productividad y capacidad de innovación crean cambios dentro de las organizaciones los cuales pueden llevar hasta a un completo nuevo modelo de negocio de la organización dependiendo si el resultado es una innovación incremental o radical. Por tal motivo es imperativo la eficiente administración al cambio soportado con el compromiso de los empleados. Las personas y los grupos son sistemas complejos adaptativos gobernados por la auto-organización no por el poder del cargo (Ehin, 2013).

\section{Bibliografía}

Bechara, A., \& Damasio, A. R. (2005). The somatic marker hypothesis: A neural theory of economic decision. Games and Economic Behavior, 52(2), 336-372. doi: http://dx.doi.org/10.1016/j.geb.2004.06.010

Becker, W. J., \& Cropanzano, R. (2010). Organizational neuroscience: The promise and prospects of an emerging discipline. Journal of Organizational Behavior, 31(7), 1055-1059.

Butts, C. T., \& Carley, K. M. (2007). Structural Change and Homeostasis in Organizations: A Decision-Theoretic Approach. Journal of Mathematical Sociology, 31(4), 295-321. doi: $10.1080 / 00222500701542517$

Cacioppo, J. T., \& Patrick, W. (2008). Loneliness : human nature and the need for social connection (1st. ed. ed.). New York ; London: W. W. Norton.

Camerer, C., Loewenstein, G., \& Prelec, D. (2005). Neuroeconomics: How Neuroscience Can Inform Economics. Journal of Economic Literature, 43(1), 9-64. doi: 10.2307/4129306 
Clark, J. V. (1962). A Healthy Organization. California Management Review, 4(4), 16-30.

Clark, T., Osterwalder, A., \& Pigneur, Y. (2012). Business model you: Pearson France.

Damasio, A. R. (2010). Self comes to mind : constructing the conscious brain (1st ed.). New York: Pantheon Books.

Ehin, C. (2009). The organizational sweet spot : engaging the innovative dynamics of your social networks. Dordrecht; New York: Springer.

Ehin, C. (2010). The duality of organizational effectiveness. Retrieved from unManagement website: http://www.unmanagement.com/work/the-duality-of-organizationaleffectiveness/

Ehin, C. (2013). Can people really be managed? International Journal of Commerce \& Management, 23(3), 184-203.

Ehin, C., \& Kivikas, T. (2012). Catalytic Leadership and Sociocultural Homeostasis. The Journal for Quality and Participation, 35(2), 31-34.

Gardner, D. G., \& Pierce, J. L. (2013). Focus of attention at work and organization-based selfesteem. Journal of Managerial Psychology, 28(2), 110-132. doi: http://dx.doi.org/10.1108/02683941311300243

Hannan, M. T., \& Freeman, J. (1977). The Population Ecology of Organizations. American Journal of Sociology, 82(5), 929-964. doi: 10.2307/2777807

Jacobs, C. S. (2009). Management rewired : why feedback doesn't work and other surprising lessons from the latest brain science. New York, N.Y.: Portfolio.

Lattuch, F., \& Young, S. (2011). Young professionals' perceptions toward organizational change. Leadership \& Organization Development Journal, 32(6), 605-627. doi: http://dx.doi.org/10.1108/01437731111161085

Martins, N. (2010). Can neuroscience inform economics? Rationality, emotions and preference formation. Cambridge Journal of Economics, 35(2), 251-267. doi: 10.1093/cje/beq017

Parkes, C., \& Borland, H. (2012). Strategic HRM: Transforming Its Responsibilities Toward Ecological Sustainability-- The Greatest Global Challenge Facing Organizations. Thunderbird International Business Review, 54(6), 811-824. doi: 10.1002/tie.21505

Theoretical Foundation for Sociocultural Homeostasis. (2012). The Journal for Quality and Participation, 35(2), 1-5. 